\title{
Positive Borders or Negative Borders: How to Make Lossless Generator-based Representations Concise
}

\author{
Guimei $\mathrm{Liu}^{1,2} \quad$ Jinyan $\mathrm{Li}^{1} \quad$ Limsoon Wong ${ }^{2} \quad$ Wynne Hsu ${ }^{2}$ \\ ${ }^{1}$ Institute for Infocomm Research, Singapore \\ ${ }^{2}$ School of Computing, National Univeristy of Singapore, Singapore
}

\begin{abstract}
A complete set of frequent itemsets can get undesirably large due to redundancy. Several representations have been proposed to eliminate the redundancy. Existing generator based representations rely on a negative border to make the representation lossless. However, negative borders of generators are often very large. The number of itemsets on a negative border sometimes even exceeds the total number of frequent itemsets. In this paper, we propose to use a positive border together with frequent generators to form a lossless representation. A set of frequent generators plus its positive border is always no larger than the corresponding complete set of frequent itemsets, thus it is a true concise representation. The generalized form of this representation is also proposed. We develop an efficient algorithm, called GrGrowth, to mine generators and positive borders as well as their generalizations.
\end{abstract}

\section{Introduction}

Frequent itemset mining is an important problem in the data mining area. It was first introduced by Agrawal et al.[1] in the context of transactional databases. The number of frequent itemsets can be undesirably large, especially on dense datasets where long patterns are prolific. Many frequent itemsets are redundant because their support can be inferred from other frequent itemsets. Generating too many frequent itemsets not only requires extensive mining cost but also defeats the primary purpose of data mining in the first place.

Several concepts have been proposed to eliminate the redundancy from a complete set of frequent itemsets, including frequent closed itemsets [12], generators [2] and generalizations of generators $[4,10]$. In some applications, generators are more preferable than closed itemsets. For example, generators are more appropriate for classification than closed itemsets because closed itemsets contain some redundant items that are not useful for classification, which also violates the minimum description length principle. The redundant items sometimes have a negative impact on classification ac- curacies because they can prevent a new instance from matching a frequent itemset.

A representation is lossless if we can decide for any itemset whether it is frequent and we can determine the support of the itemset if it is frequent, using only information of the representation without accessing the original database. Generators alone are not adequate for representing a complete set of frequent itemsets. Existing generator based representations $[4,9,10]$ use a negative border together with frequent generators to form a lossless representation. We have observed that negative borders are often very large, sometimes, the negative border alone is larger than the corresponding complete set of frequent itemsets. For example, the total number of frequent itemsets is 122450 in dataset BMS-POS with minimum support of $0.1 \%$, while the number of itemsets on the negative border is 236912. To solve this problem, we propose a new concise representation of frequent itemsets, which uses a positive border together with generators to form a lossless representation.

The main contributions of the paper are summarized as follows: (1) We propose a new concise representation of frequent itemsets, which uses a positive border instead of a negative border together with frequent generators to represent a complete set of frequent itemsets. The size of the new representation is guaranteed to be no larger than the total number of frequent itemsets, thus it is a true concise representation. Our experiment results show that a positive border is usually orders of magnitude smaller than its corresponding negative border. (2) The completeness of the new representation is proved, and an algorithm is given to derive the support of an itemset from the new representation. (3) We develop an efficient algorithm GrGrowth to mine frequent generators and positive borders. The GrGrowth algorithm and the concept of positive borders can be both applied to generalizations of generators, such as disjunction-free sets and generalized disjunction-free sets.

The rest of the paper is organized as follows. Section 2 presents related work. The formal definitions 
of positive border based representations are given in Section 3. Section 4 describes the mining algorithm. The experiment results are shown in Section 5 .

\section{Related Work}

The problem of removing redundancy while preserving semantics has drawn much attention in the data mining area. Several concepts have been proposed to remove redundancy from a complete set of frequent itemsets, including frequent closed itemsets [12], generators [2] and generalizations of generators $[4,10,5,6,3]$.

The concept of generators is first introduced by Bastide et al. [2]. Bykowski et al. [4] propose another concept - disjunction-free generator to further reduce result size. Generators or disjunction-free generators alone are not adequate to represent a complete set of frequent itemsets. Bykowski et al. use a negative border together with disjunction-free generators to form a lossless representation. Kryszkiewicz et al. [10] generalize the concept of disjunction-free generator and propose to mine generalized disjunction-free generators. Boulicaut et al. [3] generalize the generator representation from another direction and propose the $\delta$-free-sets representation. Itemset $l$ is $\delta$-free if the support difference between $l$ and $l$ 's subsets is less than $\delta$. Boulicaut et al. also use a negative border together with $\delta$-freesets to form a concise representation. The $\delta$-free-sets representation is not lossless unless $\delta=0$.

Mannila et al. [11] first propose the notion of condensed representation based on the inclusion-exclusion principle. Calders et al. [5] propose a similar conceptnon-derivable frequent itemsets. An itemset is nonderivable if its support cannot be inferred from its subsets based on the inclusion-exclusion principle. Calders et al. develop a level-wise algorithm NDI [5] and a depth-first algorithm dfNDI [7] to mine non-derivable frequent itemsets. The dfNDI algorithm is shown to be much more efficient than the NDI algorithm. Calders et al. [6] also propose the concept of $\mathrm{k}$-free sets and several types of borders to form lossless representations. However, the computation cost for inferring support from non-derivable itemsets and k-free sets is very high.

\section{Positive Border Based Representations}

In this section, we first give the formal definitions of generators and positive borders, and then give an algorithm to infer the support of an itemset from positive border based representations.

\subsection{Definitions}

Let $I=\left\{a_{1}, a_{2}, \cdots, a_{n}\right\}$ be a set of items and $D=\left\{t_{1}, t_{2}, \cdots, t_{N}\right\}$ be a transaction database, where $t_{i}(i \in[1, N])$ is a transaction and $t_{i} \subseteq I$. Each subset

of $I$ is called an itemset. The support of an itemset $l$ in $D$ is defined as support $(l)=\mid\{t \mid t \in D$ and $l \subseteq t\}|/| D \mid$ or $\operatorname{support}(l)=\mid\{t \mid t \in D$ and $l \subseteq t\} \mid$.

Definition 3.1. (Generator) Itemset $l$ is a generator if there does not exist $l^{\prime}$ such that $l^{\prime} \subset l$ and $\operatorname{support}\left(l^{\prime}\right)=\operatorname{support}(l)$.

According to the definition, the empty set $\phi$ is a generator in any database. If an itemset is a generator in a database and its support is no less than a given minimum support threshold, we call the itemset $a$ frequent generator. Generators also have the antimonotone property.

Property 3.1. (ANTI-MONOTONE PROPERTy) If $l$ is not a generator, then $\forall l^{\prime} \supset l, l^{\prime}$ is not a generator.

\begin{tabular}{|c|l|}
\hline Tid & Transactions \\
\hline 1 & $a, b, c, d, e, g$ \\
2 & $a, b, d, e, f$ \\
3 & $b, c, d, e, h, i$ \\
4 & $a, d, e, m$ \\
5 & $c, d, e, h, n$ \\
6 & $b, e, i, o$ \\
\hline
\end{tabular}

(a)

\begin{tabular}{|l|}
\hline Frequent Generators \\
\hline$\phi: 6, d: 5, b: 4, a: 3, c: 3, h: 2$ \\
$i: 2, b d: 3, a b: 2, b c: 2$ \\
\hline
\end{tabular}

(c)

\begin{tabular}{|l|}
\hline Frequent itemsets \\
\hline$\phi: 6, e: 6, d: 5, b: 4, a: 3, c: 3, h: 2$ \\
$i: 2$, ed:5, be:4, bd:3, bde:3, ae:3 \\
ad:3, ade:3 ab:2, abe:2, abd:2 \\
abde:2, ce:3, cd:3, cde:3, cb:2 \\
cbe:2, cbd:2, cbde:2, he:2, hc:2 \\
hce:2, hd:2, hde:2, hcd:2 \\
hcde:2, ie:2, ib:2, ibe:2 \\
\hline
\end{tabular}
(b)

\begin{tabular}{|l|}
\hline Positive Border \\
\hline$\langle\phi, e\rangle: 6,\langle a, d\rangle: 3,\langle c, d\rangle: 3$ \\
$\langle h, c\rangle: 2,\langle h, d\rangle: 2,\langle i, b\rangle: 2$ \\
\hline
\end{tabular}

Table 1: An example (min_sup=2)

Example. Table 1(a) shows an example transaction database containing 6 transactions. With minimum support of 2 , the set of frequent itemsets are shown in Table 1(b) and the set of frequent generators are shown in Table 1(c). For brevity, a frequent itemset $\left\{a_{1}, a_{2}, \cdots, a_{m}\right\}$ with support $s$ is represented as $a_{1} a_{2} \cdots a_{m}: s$. Many frequent itemsets are not generators. For example, itemset $e$ is not a generator because it has the same support as $\phi$. Consequently, all the supersets of $e$ are not generators.

Definition 3.2. (The POSITIVE BORDER OF $F G$ ) Let $F G$ be the set of frequent generators in a database with respect to a minimum support threshold. The positive border of $F G$ is defined as $P B d(F G)=$ $\left\{l \mid l\right.$ is frequent $\left.\wedge l \notin F G \wedge\left(\forall l^{\prime} \subset l, l^{\prime} \in F G\right)\right\}$.

Example. Table 1(d) shows the positive border of frequent generators with minimum support of 2 in the database shown in Table 1(a). We represent an itemset $l$ on a positive border as a pair $\left\langle l^{\prime}, x\right\rangle$, where $x$ is an item, $l^{\prime}=l-\{x\}$ and support $\left(l^{\prime}\right)=\operatorname{support}(l)$. For 
example, itemset $e$ is on the positive border and it has the same support as $\phi$, hence it is represented as $\langle\phi, e\rangle$. The second pair $\langle a, d\rangle$ represents itemset $a d$.

Note that for any non-generator itemset $l$, there must exist itemset $l^{\prime}$ and item $x$ such that $l^{\prime}=l-\{x\}$ and support $\left(l^{\prime}\right)=\operatorname{support}(l)$ according to the definition of generators. The itemsets on positive borders are not generators, therefore any itemset $l$ on a positive border can be represented as a pair $\left\langle l^{\prime}, x\right\rangle$ such that $l^{\prime}=l-\{x\}$ and support $\left(l^{\prime}\right)=\operatorname{support}(l)$. For itemset $l$ on a positive border, there are possibly more than one pairs of $l^{\prime}$ and $x$ satisfying that $l^{\prime}=l-\{x\}$ and $\operatorname{support}\left(l^{\prime}\right)=\operatorname{support}(l)$. Any pair can be chosen to represent $l$.

Proposition 3.1. Let FI and FG be the complete set of frequent itemsets and the set of frequent generators in a database respectively. We have $F G \bigcap P B d(F G)=\phi$ and $F G \bigcup P B d(F G) \subseteq F I$, thus $|F G|+|P B d(F G)| \leq$ $|F I|$.

This is true by the definition of frequent generators and positive borders. Proposition 3.1 states that a set of frequent generators plus its positive border is always a subset of the complete set of frequent itemsets, thus it is a true concise representation. Next we prove that this representation is lossless.

LEMMA 3.1. $\forall$ frequent itemset $l$, if $l \notin F G$ and $l \notin$ $P B d(F G)$, then $\exists l^{\prime} \in P B d(F G)$ such that $l^{\prime} \subset l$.

Proof. We prove the lemma using induction on the length of the itemsets. It is easy to prove that the lemma is true when $|l| \leq 2$.

Assume that when $|l| \leq k(k \geq 0)$, the lemma is true. Let $|l|=k+1$. The fact that $l \notin F G$ and $l \notin$ $P B d(F G)$ means that $\exists l^{\prime} \subset l$ such that $l^{\prime} \notin F G$. If $l^{\prime} \in P B d(F G)$, then the lemma is true. Otherwise by using the assumption, there must exist $l^{\prime \prime} \subset l^{\prime}$ such that $l^{\prime \prime} \in P B d(F G)$. Hence the lemma is also true because $l^{\prime \prime} \subset l^{\prime} \subset l$.

LEMмA 3.2. $\forall$ itemset $l$ and item $a$, if $\operatorname{support}(l)=$ $\operatorname{support}(l \bigcup\{a\})$, then $\forall l^{\prime} \supset \quad l, \operatorname{support}\left(l^{\prime}\right)=$ support $\left(l^{\prime} \cup\{a\}\right)$.

TheOREM 3.1. Given $F G$ and $P B d(F G)$ with support information, $\forall l$, we can determine: (1) whether $l$ is frequent, and (2) the support of $l$ if $l$ is frequent.

Proof. If $l \in F G$ or $l \in P B d(F G)$, we can obtain the support of $l$ directly.

Otherwise if there exists itemset $l^{\prime}$ such that $l^{\prime} \subset l$ and $l^{\prime} \in \operatorname{PBd}(F G)$, let $l^{\prime \prime}$ be the itemset such that $l^{\prime \prime}=l^{\prime}-\{a\}$, support $\left(l^{\prime \prime}\right)=\operatorname{support}\left(l^{\prime}\right)$ and $l^{\prime \prime} \in F G$, we have support $\left(l^{\prime \prime}\right)=\operatorname{support}\left(l^{\prime \prime} \bigcup\{a\}\right)$ and $l^{\prime \prime}=l^{\prime}-\{a\} \subset$ $l-\{a\}$. According to Lemma 3.2, we have support $(l-$ $\{a\})=\operatorname{support}(l)$. We remove item $a$ from $l$. This process is repeated until there does not exist $l^{\prime}$ such that $l^{\prime} \in P B d(F G)$ and $l^{\prime} \subset l$. The resultant itemset is denoted as $\bar{l}$, and $\bar{l}$ can be in two cases: (1) $\bar{l} \in$ $F G$ or $\bar{l} \in \operatorname{PBd}(F G)$, then $l$ must be frequent and support $(l)=$ support $(\bar{l})$ according to Lemma 3.2 ; and $(2)$ $\bar{l} \notin F G$ and $\bar{l} \notin P B d(F G)$, then $l$ must be infrequent because otherwise it conflicts with Lemma 3.1.

It directly follows from Theorem 3.1 that the set of frequent generators in a database and its positive border form a concise lossless representation of the complete set of frequent itemsets.

\subsection{Inferring support}

From the proof of Theorem 3.1, we can get an algorithm for inferring the support of an itemset from positive border based concise representations. Intuitively, if an itemset is not a generator, then the itemset must contain some redundant items. Removing these redundant items does not change the support of the itemset. Itemsets on positive borders are the minimal itemsets that contain one redundant item. We represent an itemset $l$ on a positive border as $\left\langle l^{\prime}, a\right\rangle$, where $l^{\prime}=l-\{a\}$ and $\operatorname{support}\left(l^{\prime}\right)=\operatorname{support}(l)$, so the redundant items can be easily identified. When inferring the support of an itemset, we first use positive borders to remove redundant items from this itemset. If the resultant itemset is a generator, then the original itemset is frequent and its support equals to the resultant itemset, otherwise the itemset is infrequent.

Example. To check whether itemset bcde is frequent and obtain its support if it is frequent, we first search in Table 1(d) for the subsets of bcde. We find $\langle\phi, e\rangle$, so item $e$ is removed. Then we continue the search and find $\langle c, d\rangle$. Item $d$ is removed and the resultant itemset is $b c$. We find $b c$ in Table $1(c)$. Therefore, itemset bcde is frequent and its support is 2 .

To check whether itemset $a c d h$ is frequent and obtain its support if it is frequent, we first search for its subsets in Table $1(\mathrm{~d})$. We find $\langle c, d\rangle$, so item $d$ is removed. We continue the search and find $\langle h, c\rangle$ is a subset of $a c h$, so item $c$ is removed. There is no subset of $a h$ in Table 1(d). Itemset ah does not appear in Table 1(c) either, so itemset $a c d h$ is not frequent.

\subsection{Generalizations}

We can also define positive borders for generalized forms of generators.

DEFINITION 3.3. ( $k$-DISJUNCTION-FREE SET) Itemset $l$ is a $k$-disjunction-free set if there does not exist itemset 
$l^{\prime}$ such that $l^{\prime} \subset l,|l|-\left|l^{\prime}\right| \leq k$ and support $(l)=$ $\sum_{l^{\prime} \subseteq l^{\prime \prime} \subset l}(-1)^{|l|-\left|l^{\prime \prime}\right|-1} \cdot \operatorname{support}\left(l^{\prime \prime}\right)$.

According to Definition 3.3, if an itemset is a $k$ disjunction-free set, it must be a $(k-1)$-disjunctionfree set. Generators are 1-disjunction-free sets. The disjunction-free sets proposed by Bykowski et al [4] are 2-disjunction-free set. The generalized disjunctionfree sets proposed by Kryszkiewicz et al. [10] are $\infty$ disjunction-free sets.

Example. In the example shown in Table 1, itemset $b d$ is a generator, but it is not a 2-disjunction-free set because $\operatorname{support}(b d)=-\operatorname{support}(\phi)+\operatorname{support}(b)+\operatorname{support}(d)$.

Definition 3.4. (The POSITIVE Border of $F G_{k}$ ) Let $F G_{k}$ be the set of frequent $k$-disjunction-free sets in a database with respect to a minimum support threshold. The positive border of $F G_{k}$ is defined as $P B d\left(F G_{k}\right)=$ $\left\{l \mid l\right.$ is frequent $\left.\wedge l \notin F G_{k} \wedge\left(\forall l^{\prime} \subset l, l^{\prime} \in F G_{k}\right)\right\}$.

The set of frequent $k$-disjunction-free sets $(k>1)$ in a database and its positive border also form a lossless concise representation of the complete set of frequent itemsets. The proof is similar to the proof of Theorem 3.1. We omit it here.

\section{The GrGrowth algorithm}

The GrGrowth algorithm adopts the pattern growth approach to mine frequent generators and positive borders. It constructs a conditional database for each frequent generator and uses FP-tree to store the conditional databases. The GrGrowth algorithm prunes nongenerators during the mining process to save mining cost. Generators and itemsets on positive borders are identified by checking two conditions: (1) whether all the subsets of a frequent itemset are generators, and (2) whether all the subsets of the frequent itemset are more frequent than the itemset. If a frequent itemset satisfies both conditions, then the itemset is a frequent generator; if a frequent itemset satisfies only the first condition, then the itemset is on the positive border; otherwise, the itemset should be discarded. According to the anti-monotone property of generators, only the conditional databases of frequent generators should be processed. The search space of the frequent itemset mining problem can be represented by a set-enumeration tree. The GrGrowth algorithm uses depth-first right-to-left order to traverse the set-enumeration tree to guarantee that all the subsets of a frequent itemset are discovered before that itemset. It uses a hash-table to store all the generators that have been discovered so far during the mining process to facilitate subset checking. The GrGrowth algorithm can be easily extended to mine generalizations of the positive border based representations.

\begin{tabular}{|c|c|c|c|c|c|}
\hline Datasets & Size & \#Trans & \#Items & MaxTL & AvgTL \\
\hline accidents & $34.68 \mathrm{MB}$ & 340,183 & 468 & 52 & 33.81 \\
BMS-POS & $11.62 \mathrm{MB}$ & 51,5597 & 1,657 & 165 & 6.53 \\
BMS-WebView-1 & $0.99 \mathrm{MB}$ & 59,602 & 497 & 268 & 2.51 \\
BMS-WebView-2 & $2.34 \mathrm{MB}$ & 77,512 & 3,340 & 162 & 4.62 \\
chess & $0.34 \mathrm{MB}$ & 3,196 & 75 & 37 & 37.00 \\
connect-4 & $9.11 \mathrm{MB}$ & 67,557 & 129 & 43 & 43.00 \\
mushroom & $0.56 \mathrm{M}$ & 8,124 & 119 & 23 & 23.00 \\
pumsb & $16.30 \mathrm{MB}$ & 49,046 & 2,113 & 74 & 74.00 \\
pumsb_star & $11.03 \mathrm{MB}$ & 49,046 & 2,088 & 63 & 50.48 \\
retail & $4.07 \mathrm{MB}$ & 88,162 & 16,470 & 77 & 10.31 \\
T10I4D100k & $3.93 \mathrm{MB}$ & 100,000 & 870 & 30 & 10.10 \\
T40I10D100k & $15.12 \mathrm{MB}$ & 100,000 & 942 & 78 & 39.61 \\
\hline
\end{tabular}

Table 2: Datasets

\section{A Performance Study}

The experiments were conducted on a 3.00Ghz Pentium IV with 2GB memory running Microsoft Windows XP professional. All implementations were complied using Microsoft Visual $\mathrm{C}++6.0$. Table 2 shows the datasets used in our performance study and some statistical information of these datasets. All these datasets are available at http://fimi.cs.helsinki.fi/data/.

\subsection{Border size comparison}

The first experiment is to compare the size of positive borders with that of negative borders. Table 3 shows the total number of frequent itemsets ("FI"), the number of frequent closed itemsets ("FCI"), the number of frequent generators ("FG"), the size of the negative border of $F G$ ("NBd(FG)"), the size of the positive border of $F G$ (" $\mathrm{PBd}(\mathrm{FG})$ "), the number of frequent $\infty$-disjunction-free generators ( $F G_{\infty}$ "), the size of the negative border of $F G_{\infty}\left(" \operatorname{NBd}\left(F G_{\infty}\right)\right.$ ") $)$ and the size of the positive border of $F G_{\infty}\left(" \operatorname{PBd}\left(F G_{\infty}\right)\right.$ ") on each dataset. The minimum support thresholds are shown in the second column.

The numbers in Table 3 indicates that negative borders are often significantly larger than the corresponding complete sets of frequent itemsets on sparse datasets. For example, in dataset retail with minimum support of $0.005 \%$, the number of itemsets on the negative border of $F G$ is 64914318 , which is about 43 times larger than the total number of frequent itemsets and about 585 times larger than the number of itemsets on the positive border of $F G$. The negative borders shrink little with the increase of $k$ on sparse datasets. Even with $k=\infty$, it is still often the case that negative borders are much larger than the corresponding complete sets of frequent itemsets on sparse datasets. This is unacceptable for a concise representation. On the contrary, the positive border based representations are always smaller than the corresponding complete sets of frequent itemsets, thus are true concise representations. 


\begin{tabular}{|c|c|c|c|c|c|c|c|c|c|}
\hline Datasets & min_sup & FI & FCI & $F G$ & $\operatorname{NBd}(F G)$ & $\operatorname{PBd}(F G)$ & $F G_{\infty}$ & $\operatorname{NBd}\left(F G_{\infty}\right)$ & $\operatorname{PBd}\left(F G_{\infty}\right)$ \\
\hline accidents & $10 \%$ & 10691550 & 9958684 & 9958684 & 134282 & 851 & 532458 & 77227 & 142391 \\
\hline accidents & $30 \%$ & 149546 & 149530 & 149530 & 5096 & 1 & 24650 & 4596 & 5415 \\
\hline BMS-POS & $0.03 \%$ & 1939308 & 1761608 & 1761611 & 1711467 & 57404 & 1466347 & 1690535 & 160690 \\
\hline BMS-WebView-1 & $0.05 \%$ & 485490182335 & 127132 & 485327 & 315526 & 460523 & 284640 & 282031 & 549252 \\
\hline BMS-WebView-1 & $0.1 \%$ & 3992 & 3975 & 3979 & $66629 *$ & 12 & 3971 & $66629 *$ & 19 \\
\hline chess & $20 \%$ & 289154814 & 22808625 & 25031186 & 705394 & 838 & 24769 & 6749 & 12517 \\
\hline chess & $45 \%$ & 2832778 & 705111 & 716948 & 27396 & 88 & 3347 & 1275 & 1882 \\
\hline connect-4 & $10 \%$ & 58062343952 & 8035412 & 8035412 & 175990 & 146 & 19494 & 8388 & 9676 \\
\hline connect- 4 & $35 \%$ & 667235248 & 328345 & 328345 & 11073 & 95 & 1137 & 645 & 1388 \\
\hline mushroom & $0.1 \%$ & 1727758092 & 147905 & 323432 & 78437 & 20035 & 118475 & 42354 & 30400 \\
\hline pumsb_star & $20 \%$ & 7122280454 & 122202 & 253107 & 14638 & 1625 & 39051 & 12327 & 13316 \\
\hline retail & $0.005 \%$ & 1506776 & 504143 & 532343 & $64914318 *$ & 110918 & 500814 & $64909090 *$ & 133658 \\
\hline retail & $0.01 \%$ & 240853 & 189078 & 191266 & $40565727 *$ & 13877 & 184965 & $40564812^{*}$ & 18557 \\
\hline T10I4D100k & $0.005 \%$ & 1923260 & 769778 & 994903 & $24669957 *$ & 374562 & 978510 & $24669812 *$ & 384667 \\
\hline T10I4D100k & $0.05 \%$ & 52623 & 46315 & 46751 & $678244^{*}$ & 1257 & 38566 & $678180^{*}$ & 5093 \\
\hline T40I10D100k & $1 \%$ & 65237 & 65237 & 65237 & 521359* & 0 & 33883 & $510861 *$ & 7372 \\
\hline
\end{tabular}

Bold: The lossless representation is not really concise, for example, $\|F G \cup N B d(F G)\|>\|F I\|$ or $\left\|F G_{\infty} \cup N B d\left(F G G_{\infty}\right)\right\|>\|F I\|$ * : $\|N B d(F G)\|>\|F I\|$.

Table 3: Size comparison between different representations

\subsection{Mining time}

The second experiment is to study the efficiency of the GrGrowth algorithm. We compare the GrGrowth algorithm with two algorithms. One is the FPClose algorithm [8], which is one of the state-of-the-art frequent closed itemset mining algorithms. The other is a levelwise algorithm for mining frequent generators and positive borders, which is implemented based on Christian Borgelt's implementation of the Apriori algorithm. The GrGrowth algorithm outperforms the other two algorithms consistently. In particular, it is usually one or two orders of magnitude faster than the level-wise algorithm for the same task of mining frequent generators and positive borders.

\section{References}

[1] R. Agrawal, T. Imielinski, and A. N. Swami. Mining association rules between sets of items in large databases. In Proc. of the 1993 ACM SIGMOD Conference, pages 207-216, 1993.

[2] Y. Bastide, N. Pasquier, R. Taouil, G. Stumme, and L. Lakhal. Mining minimal non-redundant association rules using frequent closed itemsets. In Proc. of Computational Logic Conference, pages 972-986, 2000.

[3] J.-F. Boulicaut, A. Bykowski, and C. Rigotti. Freesets: A condensed representation of boolean data for the approximation of frequency queries. Data Mining and Knowledge Discovery Journal, 7(1):5-22, 2003.
[4] A. Bykowski and C. Rigotti. A condensed representation to find frequent patterns. In Proc. of the 20th PODS Symposium, 2001.

[5] T. Calders and B. Goethals. Mining all non-derivable frequent itemsets. In Proc. of the 6th PKDD Conference, pages 74-85, 2002.

[6] T. Calders and B. Goethals. Minimal $k$-free representations of frequent sets. In Proc. of the rth PKDD Conference, pages 71-82, 2003.

[7] T. Calders and B. Goethals. Depth-first non-derivable itemset mining. In Proc. of the 2005 SIAM International Data Mining Conference, 2005.

[8] G. Grahne and J. Zhu. Efficiently using prefix-trees in mining frequent itemsets. In Proc. of the ICDM 2003 Workshop on Frequent Itemset Mining Implementations, 2003.

[9] M. Kryszkiewicz. Concise representation of frequent patterns based on disjunction-free generators. In Proc. of the 2001 ICDM Conference, pages 305-312, 2001.

[10] M. Kryszkiewicz and M. Gajek. Concise representation of frequent patterns based on generalized disjunctionfree generators. In Proc. of the 6th PAKDD Conference, pages 159-171, 2002.

[11] H. Mannila and H. Toivonen. Multiple uses of frequent sets and condensed representations. In Proc. of the 2nd ACM SIGKDD Conference, pages 189-194, 1996.

[12] N. Pasquier, Y. Bastide, R. Taouil, and L. Lakhal. Discovering frequent closed itemsets for association rules. In Proc. of the 7th ICDT Conference, pages 398416, 1999. 